\title{
The feasibility of obstacle awareness forwarding scheme in a visible light communication vehicular network
}

\author{
Lisa Kristiana $^{1}$, Arsyad Ramadhan Darlis ${ }^{2}$, Irma Amelia Dewi ${ }^{3}$ \\ ${ }^{1,3}$ Department of Informatics, Institut Teknologi Nasional, ITENAS, Indonesia \\ ${ }^{2}$ Department of Electrical Engineering, Institut Teknologi Nasional, ITENAS, Indonesia
}

\begin{tabular}{l} 
Article Info \\
\hline Article history: \\
Received Jan 14, 2019 \\
Revised Jun 5, 2020 \\
Accepted Jun 16, 2020 \\
\hline
\end{tabular}

Keywords:

Communication

Forwarding method

Obstacle awareness

VANET

Vehicular-to-vehicular

Visible light communication

\section{Corresponding Author:}

Lisa Kristiana,

Department of Informatics,

Institut Teknologi Nasional, ITENAS,

Jl. PKH Mustafa No 23, Bandung 40124, Indonesia.

Email: lisa@itenas.ac.id

\begin{abstract}
A vehicular-to-vehicular $(\mathrm{V} 2 \mathrm{~V})$ communication is a part of a vehicular ad-hoc network (VANET) that emerges recently due to the heavy traffic environment. $\mathrm{V} 2 \mathrm{~V}$ is a frequently changing network since it implements vehicles as mobile nodes. The challenges in implementing $\mathrm{V} 2 \mathrm{~V}$ are the relatively short duration of possible communication and the uneven city environment caused by high rise buildings or other objects that distract the signal transmission. The limited transmitting duration between vehicles requires efficient coordination and communication. This work focuses on the utility of visible light communication in vehicular network (VLC-VN) in data transmitting and the obstacle awareness in the forwarding scheme based on our knowledge in previous researches. The result of evaluating the feasibility of VLC-VN forwarding in a freeway environment the transmission delay is lower than 1 second in 500 byte data transmission, however it reaches to only about $4 \%$ in throughput as a drawback.
\end{abstract}

Copyright $(92020$ Institute of Advanced Engineering and Science. All rights reserved.

\section{INTRODUCTION}

The vehicular ad-hoc network (VANET) has been rising since the last decade due to mobile communications of a human behavior. In the modern society, people need to commute from home to working places in a daily basis. This commuting behavior requires a mobile communication system that facilitates all types of communication such as sending emails and accessing real-time traffic information while driving [1-3].

Principally, vehicular-to-vehicular (V2V) Network is a network where vehicles as moving nodes connect to each other to share and exchange information $[4,5]$. To support this, vehicles in the vehicular network can be equipped with a visible light communication (VLC) [6, 7] device where the process of sharing information and exchanging messages relies on the connection among vehicles using lights as illustrated in Figure 1 [7].

The VLC in vehicular network (VLC-VN) is one type of wireless communications that performs the flexibility in terms of time and spatial. In terms of time flexibility feature, VLC-VN can build a connection at any time without concerning about the reliable internet connection. In terms of spatial flexibility, VLC-VN can be built under any circumstances where there is no fixed internet infrastructure. However, VLC-VN can be challenging due to several reasons, on one hand the connection can be disconnected in a very short period as a disadvantage in terms of time, and on the other hand the effect of obstacles such as buildings and overpass constructions can distract the wireless signal from one vehicle as a transmitter to other vehicles as receivers [8]. 
The process to ensure the message transmission contains of several stages. The first stage is a position coordinate knowledge of each vehicle as the initiation process. These position coordinates are important to the next process: forwarding messages. In the forwarding process, a vehicle as a source mobile node locates nearest vehicles' position. By collecting several closest coordinates, the forwarding scheme decides which vehicle will be the forwarder vehicle $[9,10]$. There are several position-based forwarding schemes in VLC-VN which will be discussed in the following subsections.

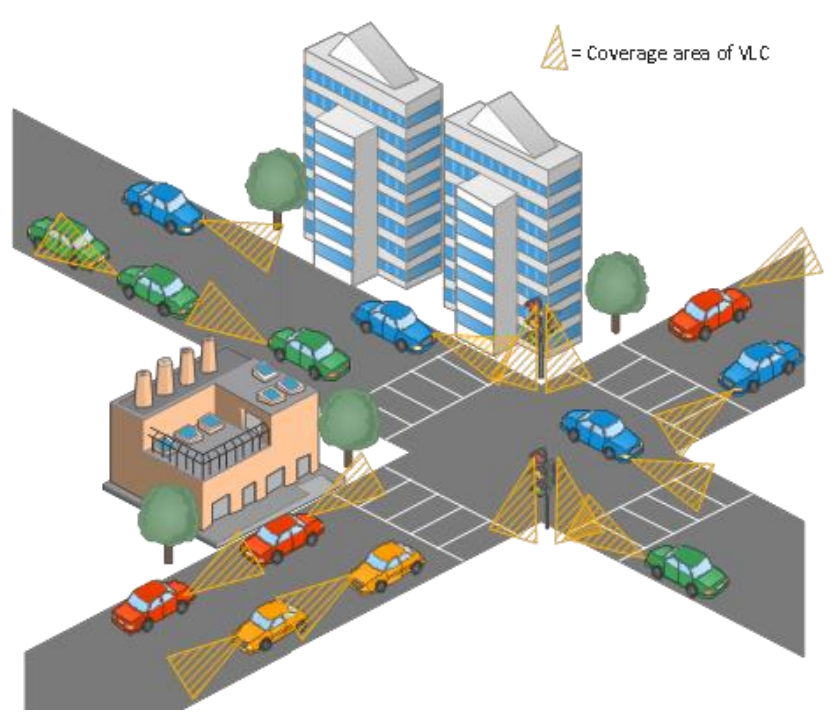

Figure 1. Visible light communication- vehicular network system

Vehicle-to-vehicular (V2V) forwarding schemes. A packet contains information is transmitted from a mobile node to another mobile node. The process of transmitting is challenging since the nodes involved are changing their position in seconds. A frequent changing position of nodes yields massive position coordinates as a function of time. The algorithm determines a current node's position by taking into account a current node's coordinates. When the current node's coordinates which can be both sender's and receiver's coordinates have tracked, the next step of the packet forwarding algorithm is to forward the packet from the sender to the receiver. This forwarding algorithm is known as a position-based forwarding scheme [11].

The advantages of the position-based forwarding scheme as follow: (1) It has a proper destination since it does not need to broadcast Hello messages in order to learn position of the other nodes. (2) It is convenient for dynamic nodes, since the forwarding algorithm updates the current node's coordinates. (3) It reduces the memory, since it does not need a full path from a sender to a destination node. (4) It improves the precision level in terms of three-dimensional environment i.e., longitude, latitude, and altitude coordinates $[12,13]$.

The Inevitable Obstacle. There are not many works done to study the impact of obstacles that interfere while transmitting the data in $\mathrm{V} 2 \mathrm{~V}$ researches $[14,15]$. In most of $\mathrm{V} 2 \mathrm{~V}$ communication researches, the existence of obstacles is often excluded in its simulation scenario. Our previous research concerns about a realistic environment in a city road [16]. The city road has several types, i.e., straight road, forked road and road with junctions. The data transmission between two vehicles has a possibility to be distracted by the obstacle in the road with junction or a crossroad. This distraction can be caused by high rise buildings and road construction as illustrated in Figure 2. In addition, the obstacle indicates a non-vehicle object such as a portal gate and a tree. The impact of the obstacle to a VLC transceiver leads to forwarding issues in transferring data [17]. Thus, this proper forwarding scheme is required. In case of a freeway environment, an unexpected heavy-duty vehicle such as a container truck also impacts the data transmission between two vehicles.

This paper is structured as follows: Section 1 introduces the challenge of VLC-VN as the alternative to wireless communication in VANET. Section 2 covers the proposed forwarding scheme in V2V which applies inevitable obstacles. Section 3 describes the proposed forwarding scheme in VLC-VN with obstacle awareness measured by an object identifier [18]. Section 4 evaluates the forwarding scheme based on real measurement that is fed into a simulation. Finally, conclusion and future work are described in section 5. 


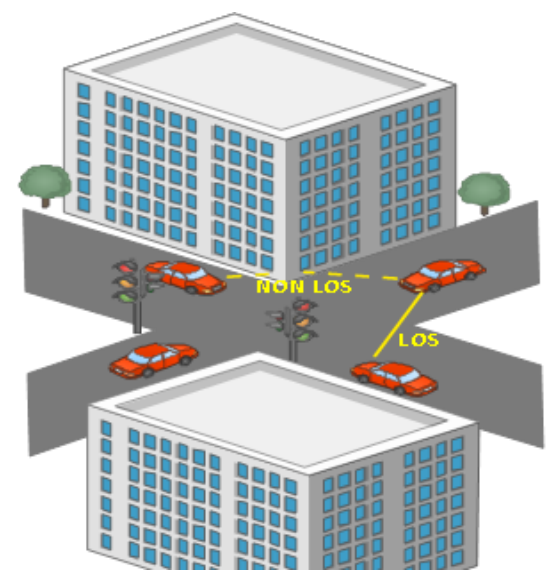

Figure 2. Data transmission over the obstacle distraction

\section{VISIBLE LIGHT COMMUNICATION VEHICULAR NETWORK (VLC-VN) FORWARDING METHOD}

Visible light communication (VLC) has been applied in wide fields such as undersea [19], and V2V communication [20, 21]. In VLC system, LED is commonly used as a light source [22] where its basic geometry is shown in Figure 3. The reflection shows field of view denoted as (FOV) on a line-of-sight (LOS) environment. That basic geometry of VLC leads to a transmission limitation in terms of reflection and Non-LOS cases due to building constructions as illustrated in Figure 2. In addition, VLC transmission has a similarity to an angle- based forwarding since it does not transmit as the other wireless transmission system such as in WiFi. Therefore, an approach to obtain the data transmission in $\mathrm{V} 2 \mathrm{~V}$ network is derived in three key points as discussed in the following subsections:

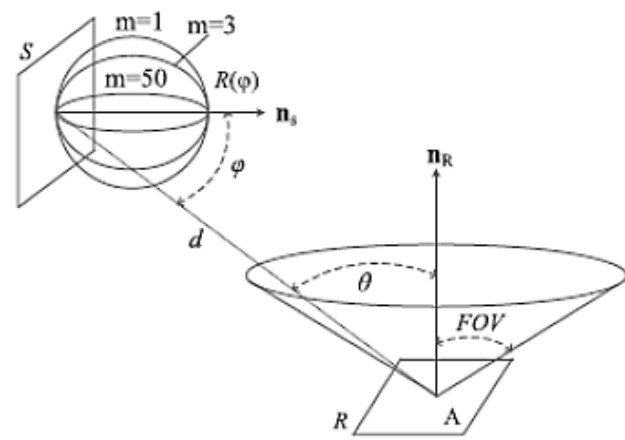

Figure 3. Basic geometry of visible light communication source, reflector and receiver [21]

\subsection{Initiation}

The first step of the VLC-VN algorithm is to initiate the connection to other closest vehicles by indicating the strongest VLC signal reception. This step is done by multicasting hello-messages to potential vehicles. The hello-message contains of coordinate position of a current vehicle, and time stamps. This initiation system obtains replies from any contacted vehicles. Vehicles located closed to the vehicle that multicasts the hello-message, replies with an acknowledgement-ACK, its coordinate location and time stamps. The reply message calculation occurs in this step by generating a hello-reply list.

\subsection{Obstacle awareness}

The second step is where the object is identified using the camera to obtain the full dimension of whether it is a vehicle or a non-vehicle. On one hand, when an object is identified as a vehicle, the obstacle awareness algorithm records the coordinate position of current vehicle. These coordinate positions are stored in a temporary table of each communicating vehicle. On the other hand, when an object is identified as a non-vehicle, the algorithm discards any information regarding to this object. Thus, the algorithm continues 
to detect other objects. This scheme is also useful for the recovery step when the data interchanging fails. This recovery step recollects the current position coordinates of communicating vehicles and recounts the possible obstacle.

\subsection{Forwarding process}

The third step is the forwarding process that consists of the forwarding decision and the forwarding step. The forwarding decision is the process where a vehicle replies with a fastest hello-reply message is selected as the next forwarder. The hello-reply message consists of location coordinates and time stamps indicating transmitted and received time. The next step is called the forwarding step where the data packet is ready to transmit based on time and distance calculations. The VLC-VN forwarding algorithm applying those three key points is represented in Figure 4.

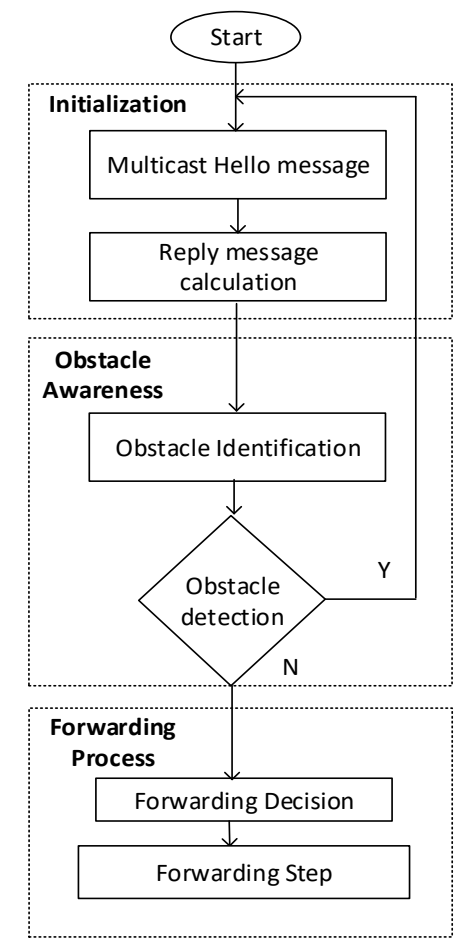

Figure 4. The VLC-VN forwarding algorithm

Since the VLC performs better in a line-of-sight (LOS) environment, one parameter regarding to the signal-loss which is affected by distance or obstacles, is considered. The negative value of this parameter indicates the non-line-of-sight (NON-LOS) situation where the obstacle is identified. In this research, that parameter is integrated in a maximum distance of VLC transmission.

\section{RESULTS AND ANALYSIS}

Vehicles are equipped with an object detector in order to identify other vehicles. Thus, other objects that are not identified as vehicles are discarded. In addition, the VLC transceiver is installed on each vehicle in order to initiate the communication. The mobility of all connecting vehicles is assumed to move on the road in high traffic density and following the freeway mobility, thus, the maximum velocity is $10-20 \mathrm{~km} / \mathrm{hr}$. The data transmission model performs the vehicle communication. Consequently, the transmission period is short and fast. VLC transceiver has been assembled as shown in Figure 5 to obtain the real measurement.

This VLC transceiver measurement defines the actual distance between two points, i.e., transmission and receiver point. This transceiver is installed on each communicating vehicle. In addition, an object identifier is installed to detect other vehicles precisely as the initial prototype of simulating V2V network. Therefore, it performs the identification process as shown in Figure 6. 


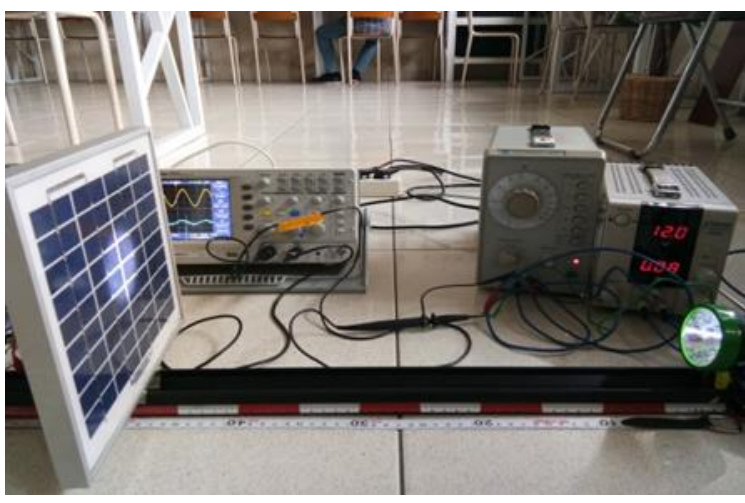

Figure 5. VLC transceiver testing

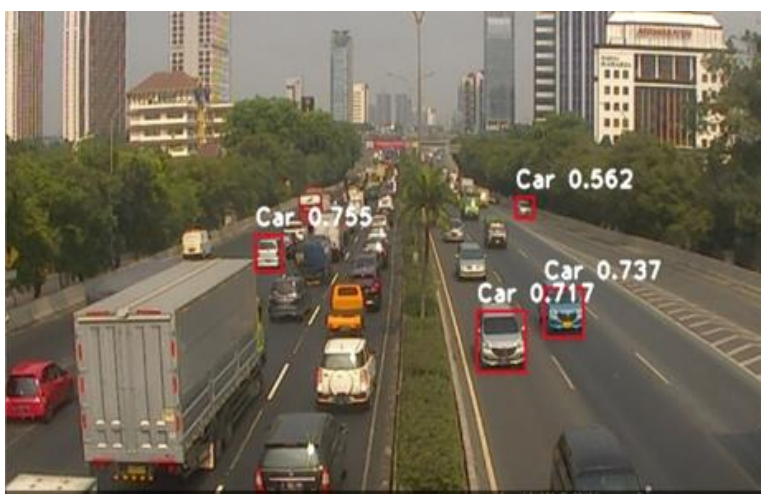

Figure 6. The output of object identifier

\subsection{Scenario and simulation setting}

Due to the simplification of VLC transceiver prototype, the simulation is conducted based on several parameters as shown in Table 1, using network simulator 3 (NS3) and SUMO for mobility [23-25]. The VLC transmission ranges up to 8 meters using $5 \mathrm{~mm}$ LED based on our experiment. The number of vehicles range from 10 to 50 vehicles where pairs of vehicles are randomly selected as the original transmitter and the destination. For example, in the 10-simulated vehicles, there are 5 pairs of vehicles that communicate each other. The transfer rate is $100 \mathrm{Mbps}$ indicates the maximum rate with the constant bit rate (CBR) data rate type. The simulation takes $300 \mathrm{~s}$ in a 500x500 meter square in a freeway environment, thus, the speed range up to maximum $50 \mathrm{~km} / \mathrm{h}$ in a high dense traffic. Finally, we apply the Greedy [26-28] based with VLC-VN forwarding scheme and run the simulation for 10 times.

Table 1. Parameter setting

\begin{tabular}{ll}
\hline Parameter & Unit \\
\hline VLC Transmission Range & $8 \mathrm{~m}$ \\
Number of Mobile Nodes & $10,20,30,40,50$ vehicles \\
Transfer rate & $100 \mathrm{Mbps}$ \\
Simulation Area & $500 \times 500 \mathrm{~m}^{2}$ \\
Simulation Time & $300 \mathrm{~s}$ \\
Vehicle's Velocity & $10 \mathrm{~km} / \mathrm{h}-50 \mathrm{~km} / \mathrm{h}$ \\
Packet Size & $500 \mathrm{~B}, 1 \mathrm{~KB}, 2 \mathrm{~KB}$ \\
Forwarding scheme & Greedy based, VLC-VN \\
Data Rate Type & Constant Bit Rate \\
Transmission Model & End-to-end \\
Mobility Model & Freeway mobility \\
\hline
\end{tabular}

\subsection{Evaluation of VLC-VN packet forwarding}

The first evaluation based on the parameter setting is the receiving hello-message packet, i.e., 20 byte. This hello-message is multicates to every mobile node that located in VLC's transmission range for 300 seconds. The number of received hello packets on respected mobile nodes shows the achievable of other mobile nodes that can connect to the source mobile node. As shown in Figure 7, it is practically reasonable to evaluate with the bigger packet size since $98 \%$ packets are received out of total 40 packets transmitted.

The second results of simulation show the size of data packet evaluation. The constant data rate of $100 \mathrm{Mbps}$ is transferred from one mobile node to another mobile node. The number of mobile nodes range from 10 to 50 nodes. This data throughput ranges from 2 to $3.8 \%$ on average as shown in Figure 8 . These data throughputs in all data packet transmissions i.e., 500B, $1 \mathrm{~KB}$ and $2 \mathrm{~KB}$ are below $5 \%$ due to the frequent connection changing between mobile nodes. This frequent connection changing leads to short duration of connection. The size of data packet does not have a significant impact to data throughput in overall simulation. In addition, the data throughputs in each simulation increase from 2.28 to $3.65 \%$ when the number of mobile node increases. This increment occurs due to the increased number of connections between mobile nodes. However, the highest amount of data throughput is achieved by transmitting 500 bytes of data and involving 40 mobile nodes. This can be concluded that the $500 \times 500$ simulation area is compatible for communicating the 40 mobile nodes which are running in maximum speed of $20 \mathrm{~km} / \mathrm{hr}$. 


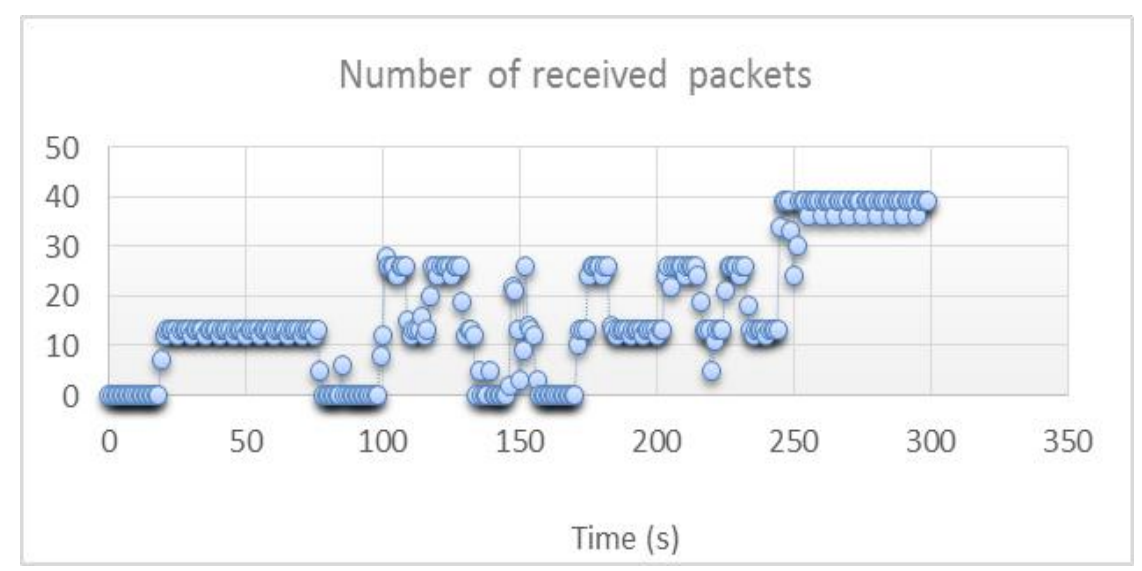

Figure 7. Hello-message packet
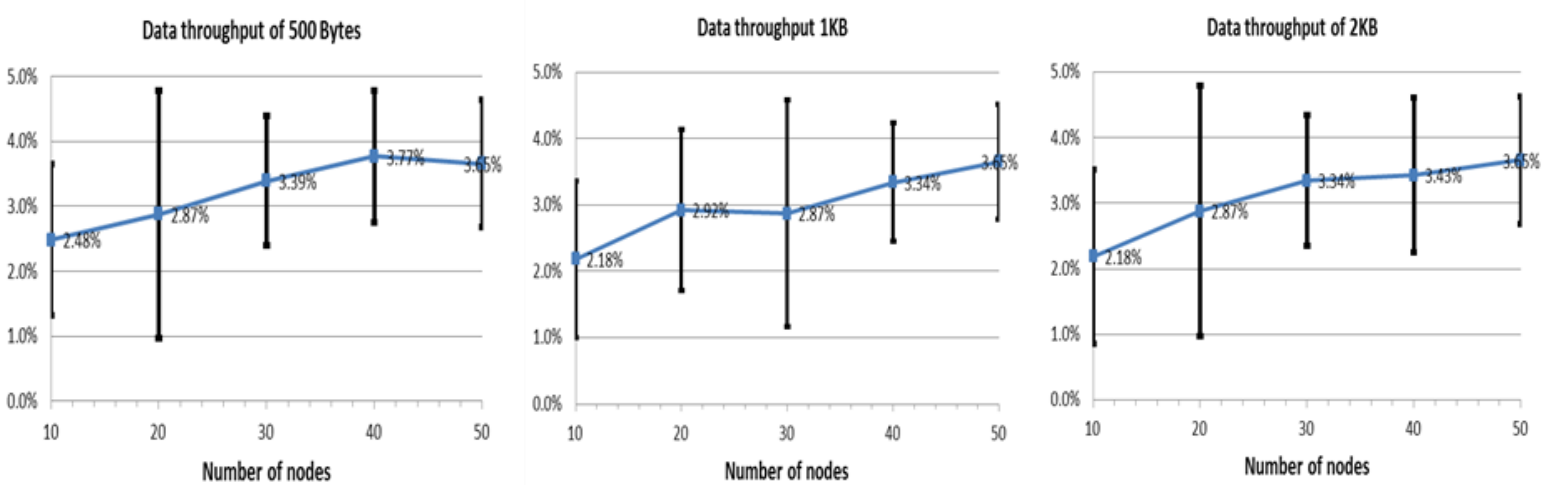

Figure 8. Throughput of $500 \mathrm{MB}, 1 \mathrm{~KB}$ and $2 \mathrm{~KB}$ of data packet

The third results of simulation show the transmission delay in all data packet transmissions i.e. $500 \mathrm{~B}, 1 \mathrm{~KB}$, and $2 \mathrm{~KB}$ is shown in Figure 9. Although the low data throughput is considered as a drawback, however, the transmission delay shows the expected end-to-end delay i.e., lower than 4 seconds, when the numbers of mobile nodes reach 40 vehicles. When there are 30 mobile nodes transmitting, the end-to-end delay reaches below 1 second. The transmission delay increases up to 15 seconds when transmitting $2 \mathrm{~KB}$ of data.
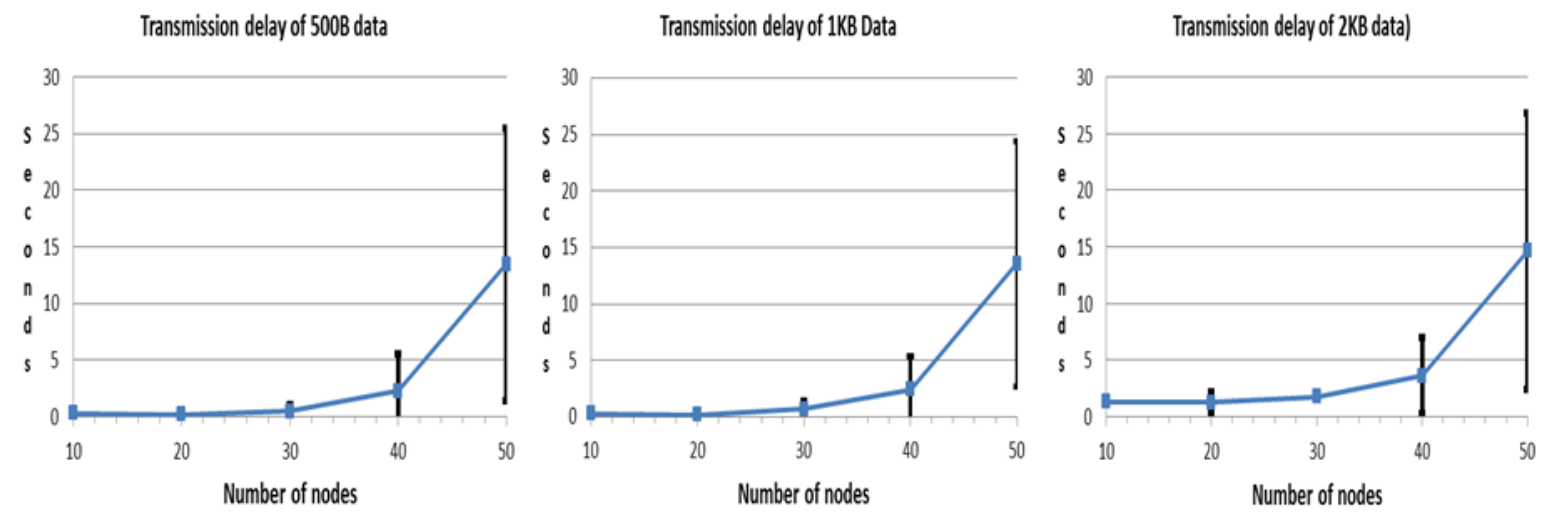

Figure 9. Transmission delay of 500B, $1 \mathrm{~KB}$, and $2 \mathrm{~KB}$ data packet transmissions 


\section{CONCLUSION AND FUTURE WORK}

This research performed the feasibility of VLC in V2V communication by applying the VLC-VN algorithm. The VLC-VN algorithm was designed to overcome the inevitable obstacle, fast and short communication between vehicles, and the VLC compatibility in a vehicular network. The results showed the low transmission delay, i.e., less than 1 second. Thus, the VLC-VN forwarding scheme can be considered as a real time data transmission in the freeway environment. As a drawback, only $3 \%$ of possible transmitting data packet in the freeway environment which is mostly caused by non-vehicle detected objects and the short transmission duration. To conclude, VLC-VN is suitable on the data transmission when the scenario is in a platooning one and in a point-to-point communication. Based on the result, the future work of VLC-VN communication will be developed by simulating a Road-side Units (RSU) as a static transceiver in order to extend the transmission duration and to avoid the impact of unexpected obstacles. In addition, the RSU is usually available on the side of every main road in a city environment, thus it can be useful not only as the traffic light, but also as the data transmitter.

\section{ACKNOWLEDGEMENTS}

This research is fully funded by The Ministry of Research and Education of the Republic of Indonesia in Hibah Penelitian Terapan Unggulan Perguruan Tinggi (PTUPT).

\section{REFERENCES}

[1] K. N. Qureshi, et al., "Road Aware Geographical Routing Protocol Coupled With Distance, Direction and Traffic Density Metrics for Urban Vehicular Adhoc Networks," Wireless Personal Communications, vol. 92, no. 3, pp. 1251-1270, 2017.

[2] S. Palaniapan and M. A. Kollathodi, "Real Time Implementation of Embedded Devices as a Security System in Intelligent Vehicles Connected via Vanets," International Journal of Electrical and Computer Engineering, vol. 9 , no. 6, pp. 4788-4797, 2019.

[3] S. Shalini and A. P. Patil, "Survey of Hybrid VANET Design for Provisioning Infotainment Application," 2019 1st International Conference on Advances in Information Technology (ICAIT), pp. 140-145, 2019.

[4] L. Kristiana, et al., "Investigating a Reliable Inter-vehicle Network in a Three-dimensional Environment," Fachgespräch in Vehicular Communication, Ulm, Germany, pp. 16-20, 2015.

[5] L. Kristiana, et al., "Evaluation of inter-vehicle connectivity in three-dimensional cases," 2017 Wireless Days, pp. 176-179, 2017.

[6] W. H. Shen and H. M. Tsai, "Testing Vehicle-to-Vehicle Visible Light Communications in Real-World Driving Scenarios,” 2017 IEEE Vehicular Networking Conference (VNC), pp. 187-194, 2017.

[7] K. Siddiqi, et al., "Visible Light Communication for V2V Intelligent Transport System," International Conference on Broadband Communications for Next Generation Networks and Multimedia Applications (CoBCom), Graz, Austria, pp. 1-4, 2016.

[8] L. Kristiana, et al., "An Analysis of Distance Extension Method in Visible Light Communication (VLC) Performance," ELKOMIKA: Jurnal Teknik Energi Elektrik, Teknik Telekomunikasi, \& Teknik Elektronika, vol. 8, no. 1 , pp. 218-228, 2020

[9] J. T. Tsai and Y. S. Han, "Alternative forwarding strategies for geographic routing in wireless networks," International Journal of Ad Hoc and Ubiquitous Computing, vol. 27, no. 4, pp. 295-307, 2018.

[10] K. Husain, A. Awang, "A receiver-based forwarding scheme to minimize multipath formation in VANET," Vehicular Ad-Hoc Networks for Smart Cities, Springer, pp. 15-26, 2017.

[11] L. Kristiana, et al., "Survey of Angle-based Forwarding Methods in VANET Communications," 2016 Wireless Days (WD), Toulouse, France, pp. 1-3, 2016.

[12] Y. He, C. Li, X. Han, Q. Lin. "A link state aware hierarchical road routing protocol for 3D scenario in VANETs," International Conference on Internet of Vehicles, Springer, Cham, pp. 11-20, 2014.

[13] L. Kristiana, et al., "Application of an Enhanced V2VUNet in a Complex Three-dimensional Inter-vehicular Communication Scenario," IEEE Asia Pacific Conference on Wireless and Mobile (APWiMob), pp. 122-127, 2017.

[14] A. Ullah, et al., "Advances in position based routing towards ITS enabled FoG-oriented VANET-A survey," IEEE Transactions on Intelligent Transportation Systems, vol. 21, no. 2, pp. 828-840, 2019.

[15] A. A. Almohammedi, et al., "Evaluating the Impact of Transmission Range on the Performance of VANET," International Journal of Electrical and Computer Engineering, vol. 6, no. 2, pp. 800-809, 2016.

[16] L. Kristiana, et al., "The Evaluation of the V2VUNet Concept to Improve Inter-vehicle Communications," International Conference on Autonomous Infrastructure, Management, and Security, pp. 94-107, 2017.

[17] L. Kristiana, et al., "A Filtering Concept for Improving the Angle-based Forwarding Scheme in Vehicular Ad-hoc Network," 22nd Asia-Pacific Conference on Communications 2016 (APCC 2016), Yogyakarta, Indonesia, pp. 545-551, 2016.

[18] I. A. Dewi, et al., "Deep Learning RetinaNet based Car Detection for Smart Transportation Network," ELKOMIKA: Jurnal Teknik Energi Elektrik, Teknik Telekomunikasi, \& Teknik Elektronika, vol. 7, no. 3, pp. 570-584, 2019. 
[19] A. R. Darlis, et al., "Shore-to-Undersea Visible Light Communication," Wireless Pers Communication, vol. 99, pp. 681-694, 2018.

[20] A. R. Darlis, "Bidirectional Underwater Visible Light Communication," International Journal of Electrical And Computer Engineering (IJECE), vol. 8, no. 6, pp. 5203-5214, 2018.

[21] Y. Qiu, et al., "Channel Modeling for Visible Light Communications—a Survey," Wireless Communication and Mobile Computing, vol. 16, no. 14, pp. 2016-2034, 2016.

[22] S. Arnon, "Visible light communication," Cambridge University Press, 2015.

[23] "Network Simulator 3 (NS3)," 2019. [Online], Available: https://www.nsnam.org.

[24] "Simulation of Urban Mobility," 2019. [Online], Available: http://sumo.sourceforge.net/.

[25] S. Mallissery, et al., "Online and Offline Communication Architecture for Vehicular Ad-hoc Networks using NS3 and SUMO Simulators," Journal of High Speed Networks, vol. 25, no. 3, pp. 253-271, 2019.

[26] H. Park, et al., "A Road Condition-based Routing and Greedy Data Forwarding Algorithm for VANETs," Adhoc \& Sensor Wireless Networks, vol. 33, no. 1, pp. 301-319, 2016.

[27] D. Wu, et al., "A Geographic Routing Protocol Based on Trunk Line in VANETs," Cyberspace Data and Intelligence, and Cyber-Living, Syndrome, and Health, Springer, Singapore, pp. 21-37, 2019.

[28] T. Nebbou, et al., "An Urban Location Service for Vehicular Area Networks," Concurrency and Computation: Practice and Experience, vol. 31, no. 24, pp. e4693, 2018.

\section{BIOGRAPHIES OF AUTHORS}
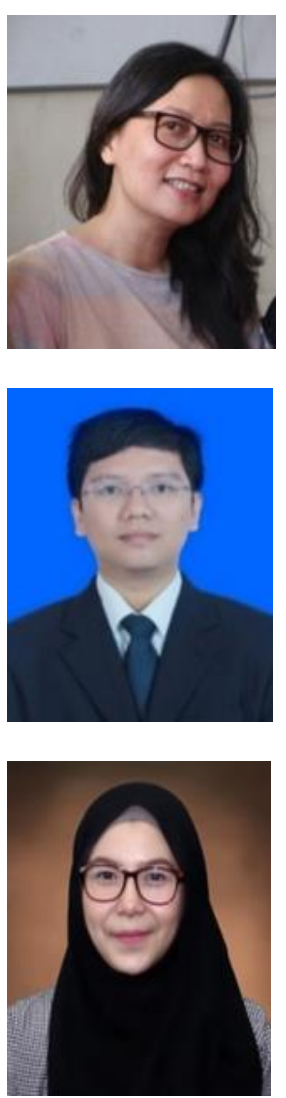

Lisa Kristiana holds a Ph.D. in Computer Science and Informatics at Business, Economics and Informatics Faculty, University of Zurich (UZH), Zurich, Switzerland. Her research interests are Mobile Communication Systems, Ad-hoc Network, Vehicular and Flying Object Network. She works as a researcher and lecturer in Department of Informatics, Institut Teknologi Nasional, Bandung, Indonesia.

Arsyad Ramadhan Darlis received a B.Sc. on Electrical Engineering from Institut Teknologi Nasional Bandung, Indonesia in 2009 and a M.Sc. on Telecommunication Engineering from Institut Teknologi Bandung (ITB), Indonesia, in 2011, respectively. His research interests are Visible Light Communications, Digital Signal Processing, and Internet-of-Things. $\mathrm{He}$ is currently a lecturer in Institut Teknologi Nasional Bandung.

Irma Amelia Dewi received a B.Sc. on Informatics Engineering from Institut Teknologi Nasional Bandung, Indonesia in 2010 and a M.Sc. on Computer Engineering from Institut Teknologi Bandung (ITB), Indonesia, in 2013. Her research interests are Digital Image Processing, Computer Vision and Artificial Intelligence. She works as a lecturer in Department of Informatics, Institut Teknologi Nasional, Itenas, Bandung. 